\title{
TWO-SITE CORRELATIONS IN A SURFACE LAYER ADSORBED ON THE CRYSTAL*
}

\author{
L. WoJTCZAK, I. ZASADA \\ Institute of Physics, Łódź University, 90-236 Łódź, Poland \\ J.C. Le Bossé, J. Lopez and J. Rousseau \\ Laboratoire de Physique des Interfaces, Bât. 203, Université Claude Bernard, \\ F-69622 Villeurbanne Cedex, France \\ (Received March 21, 1991)

\begin{abstract}
The incohorent scattering of electrons by layer adsorbed at a single crystal surface is determined by the Fourier transform of correlation functions of elements forming this layer. The model of the description of atoms or molecules adsorbed on the surface is formulated by the occupation operators which are represented by the pseudospin operators. The calculations of the correlation functions are performed by means of methods which consider a given pair of elements embedded in the effective field of remaining elements of the system while the interaction between the elements of the pair are taken in its exact form. Two approaches are presented here, i.e. the cumulant average and constant coupling approximations, and the case of binary chemisorption is considered in detail. The problem of the correlation symmetry is also discussed.
\end{abstract}

PACS numbers: 68.35.-p

\section{Introduction}

One of the most important characteristics of a surface layer is the correlation function describing some features of a crystallographic structure with respect to different kinds of disorder. The correlations reflect the statistical distribution of elements at the surface (e.g. adsorbed atoms, molecules, impurities, defects) and determine the cross sections for the scattering of particles (e.g. electrons, neutrons,

* Supported by the Project CPBP 01.08. 
$\mathrm{X}$-rays) as well as the structural factors which can appear in the expression for absorption and emission processes.

In particular, the diffuse low energy electron diffraction (DLEED) is an ilustrative example when the knowledge of correlation functions in the adsorbed layer is required. This example was considered earlier [1] by means of the methods based on the molecular field approximation and the Green function technique.

The methods presented there [1] take into consideration the interaction between one element of a system and an effective field produced by the rest of this system. Usually, the correlations however refer at least to two positions of the lattice sites and depend on the states of two elements appearing in the considered sites situated at the distance $R$. It means that the correlations are a typical example of the case when the characteristics result from the two-body interaction. Therefore, the model assuming the pairs of elements embedded in an effective field seems to be more suitable in order to reflect the natural properties of correlations. There is a reason that the discussion of various methods in two-site cluster approximation is presented here as a continuation of our considerations for correlation functions reported in the previous paper [1]. The correlation functions found for this approximation are applied to calculate the DLEED intensities for the complex substrate with the disordered overlayer.

For the present considerations we use the model of the adsorbed layer proposed by Le Bossé et al. [2] and utilized by us in [1]. The form of the adsorbate plus substrate Hamiltonian for this model is

$$
\mathcal{H}=-\sum_{p=1}^{p_{\max }} \sum_{j=1}^{N} E_{j}^{\mathrm{ads}}(p) \sigma_{j}(p)+\frac{1}{2} \sum_{p, p^{\prime}=1}^{p_{\max }} \sum_{j, j^{\prime}=1}^{N} U_{j j^{\prime}}\left(p, p^{\prime}\right) \sigma_{j}(p) \sigma_{j^{\prime}}(p) .
$$

Hamiltonian $\mathcal{H}$ depends on the occupation operators $\sigma_{j}(p)$, which characterize state $p$ of a site occupancy $j$ on the surface crystallographic lattice. It contains the quadratic term with respect to $\sigma_{j}(p)$ responsible for the interaction of the pairs of elements with the force constance $U_{j j^{\prime}}\left(p, p^{\prime}\right)$ and linear term with respect to $\sigma_{j}(p)$ describing the interaction between the adsorbate elements and the substrate with the adsorption energy $E_{j}^{\text {ads }}(p) . N$ is a number of all elements of the adsorbed layer, $\left(p_{\max }+1\right)$ is a number of all occupation states of elements.

The correlation function is defined as usual

$$
\gamma_{j j^{\prime}}\left(p, p^{\prime}\right)=\left\langle\sigma_{j}(p) \sigma_{j^{\prime}}\left(p^{\prime}\right)\right\rangle-\left\langle\sigma_{j}(p)\right\rangle\left\langle\sigma_{j^{\prime}}(p)\right\rangle
$$

and it allows us to determine an incoherent intensity of LEED which is of the form [2]

$$
\begin{aligned}
& I_{\text {incoh }}\left(k_{\mathrm{f}}^{-} \leftarrow k_{\mathrm{i}}^{+}\right)=\frac{4 \pi^{4} k}{A k_{\perp}} \sum_{p=1}^{p_{\max }} \sum_{p^{\prime}=1}^{p_{\max }} \Gamma\left(p, p^{\prime}, k_{\mathrm{f}}^{-} \leftarrow k_{\mathrm{i}}^{+}\right) \\
& \times \bar{K}_{1}^{(0)}\left(p, k_{\mathrm{f}}^{-} \leftarrow k_{\mathrm{i}}^{+}\right) K_{1}^{(0)}\left(p^{\prime}, k_{\mathrm{f}}^{-} \leftarrow k_{\mathrm{i}}^{+}\right),
\end{aligned}
$$

where $k_{i}^{-}$is a final state of wave vector $k, k_{i}^{+}$is an initial state of this wave vector, $K_{1}^{(0)}(p)$ describes the transition matrix of the $p$ adscatterer located at 
the reference site in the presence of the other adscatterers and of the substrate surface and $\Gamma\left(p, p^{\prime}, k_{\mathrm{f}}^{-} \leftarrow k_{i}^{+}\right)$is the Fourier transform of the correlation function $\gamma_{j j^{\prime}}\left(p, p^{\prime}\right)$ i.e.

$$
\Gamma\left(p, p^{\prime}, k_{\mathrm{f}}^{-} \leftarrow k_{\mathrm{i}}^{+}\right)=\frac{1}{N} \sum_{j^{\prime}, j=1}^{N} \gamma_{j j^{\prime}}\left(p, p^{\prime}\right) \exp \left[-\mathrm{i}\left(k_{\mathrm{f}}^{-}-k_{\mathrm{i}}^{+}\right)\left(T_{j^{\prime}}-T_{j}\right)\right],
$$

where the translational symmetry in the plane of adsorbed layer is assumed; all sites $j$ as the sites of correlation centers are equivalent.

In our previous paper we have shown the possibility of expression of the occupation operators $\sigma_{j}(p)$ by the pseudospin operators, which allows us to use the well-known technique of calculating the correlation functions in Ising model. Substituting the relation:

$$
\sigma_{\mathrm{j}}(p)=\sum_{n=1}^{2 S} a_{n}^{p}\left(S_{j}^{z}\right)^{n}
$$

to the IIamiltonian (1.1) and the expression (1.2) for correlation functions we have

$$
\mathcal{H}=-\sum_{j=1}^{N} \sum_{n=1}^{2 S} h_{j}^{(n)}\left(S_{j}^{z}\right)^{n}+\frac{1}{2} \sum_{j, j^{\prime}=1}^{N} \sum_{n=1}^{2 S} \sum_{n^{\prime}=1}^{2 S} U_{j j^{\prime}}^{n n^{\prime}}\left(S_{j}^{z}\right)^{n}\left(S_{j^{\prime}}^{z}\right)^{n^{\prime}}
$$

where

$$
h_{j}^{(n)}=\sum_{p=0}^{p_{\max }} E_{j}^{\text {ads }}(p) a_{n}^{p}
$$

and

$$
U_{j j^{\prime}}^{n n^{\prime}}=\sum_{p=0}^{p_{\max }} \sum_{p^{\prime}=0}^{p_{\max }} U_{j j^{\prime}}\left(p, p^{\prime}\right) a_{n}^{p} a_{n^{\prime}}^{p^{\prime}}
$$

play the role of external fields of the power $(n)$ and effective interactions of the power $\left(n+n^{\prime}\right)$ with respect to the operator $S_{j}^{z}$, respectively. The coefficients $a_{n}^{p}$ are determined by the system of equations

$$
\left(S_{j}^{z}\right)^{n}=\sum_{p_{j}=0}^{p_{\max }} \sum_{n^{\prime}=1}^{2 S} a_{n^{\prime}}^{p}\left(S_{j}^{z}\right)^{n^{\prime}}\left\langle S_{j}^{z}\left(p_{j}\right)^{n}\right\rangle
$$

and they are collected in our paper [1] for several values of pseudospin $S$.

Taking into account the above results we can write the correlation functions (1.2) in the following form:

$$
\gamma_{j j^{\prime}}\left(p, p^{\prime}\right)=\sum_{n, n^{\prime}} a_{n}^{p} a_{n^{\prime}}^{p^{\prime}} \gamma_{j j^{\prime}}^{n n^{\prime}}\left(p, p^{\prime}\right)
$$

where

$$
\gamma_{j j^{\prime}}^{n n^{\prime}}\left(p, p^{\prime}\right)=\left[\left(\left(S_{j}^{z}\right)^{n}\left(S_{j^{\prime}}^{z}\right)^{n^{\prime}}\right\rangle-\left\langle\left(S_{j}^{z}\right)^{n}\right\rangle\left\langle\left(S_{j^{\prime}}^{z}\right)^{n^{\prime}}\right\rangle\right]
$$


and the average values are calculated with respect to the Hamiltonian (1.6). In order to calculate the correlation function $\gamma_{j j^{\prime}}\left(p, p^{\prime}\right)$ we should know the correlation functions (1.9) of all the orders $n, n^{\prime} \in(0,2 S)$ for the pseudospin operators representing the occupancy of the considered sites.

Of course, all the average values appearing in the correlation functions are taken with respect to the grand canonical ensemble i.e. the statistical operator is expressed by the Hamiltonian $\tilde{\mathcal{H}}$ which relates to the chemical potential term as:

$$
\tilde{\mathcal{H}}=\mathcal{H}-\sum_{n, j} \mu_{j}^{(n)}\left(S_{j}^{z}\right)^{n}
$$

with the Hamiltonian $\mathcal{H}$ given by (1.6). Then the proper choice of $\mu_{j}^{(n)}$ assures the relations between the average values of the occupation operators, and in consequence, the pseudospin operators $\left\langle\left(S_{j}^{z}\right)^{n}\right\rangle$ and the parameters $\theta$ characterizing the degree of coverage.

The calculation of the correlation functions (1.9) for the systems described by the Hamiltonian (1.10) can be performed by means of methods which consider a given pair of elements embedded in the effective field of remaining elements of the system while the interaction between the elements of the pair are taken in its exact form. Since there are different ways to choose the effective Hamiltonian, we describe different approaches separately taking into account the most representative methods, like the cumulant average as well as constant coupling approximations.

Of course, it is possible to combine the various methods together and to calculate the correlations efficiently. For example, the generalized susceptibility can be formed on the basis of the order parameter $\left\langle S_{j}^{z}\right\rangle$ obtained by means of constant coupling approach. In this case the procedure is not new but it is equivalent to the molecular field approximation with the coefficients found more precisely.

\section{Cumulant average approach to two-site correlations}

The starting point of the cumulant average approach to the calculations of the two-site correlations is related to the average values of the operators $\left(S_{j}^{z}\right)^{n}$ and $\left(S_{j}^{z}\right)^{n}\left(S_{j}^{z}\right)^{n^{\prime}}$ for $n=1,2 \ldots 2 S$ taken with respect to the grand canonical ensemble, namely

$$
\left\langle\left(S_{j}^{z}\right)^{n}\right\rangle=\frac{\operatorname{Tr}\left[\left(S_{j}^{z}\right)^{n} \exp (-\beta \tilde{\mathcal{H}})\right]}{\operatorname{Tr} \exp (-\beta \tilde{\mathcal{H}})}
$$

and

$$
\left\langle\left(S_{j}^{z}\right)^{n}\left(S_{j^{\prime}}^{z}\right)^{n^{\prime}}\right\rangle=\frac{\operatorname{Tr}\left[\left(S_{j}^{z}\right)^{n}\left(S_{j^{\prime}}^{z}\right)^{n^{\prime}} \exp (-\beta \tilde{\mathcal{H}})\right]}{\operatorname{Tr} \exp (-\beta \tilde{\mathcal{H}})}
$$

with the Hamiltonian $\tilde{\mathcal{H}}$ given by (1.10). The approach consists in the replacement of the Hamiltonian $\tilde{\mathcal{H}}$ by its effective form which includes all the interactions 
between the considered site $j$ and its nearest neighbours $j^{\prime \prime}$ in the exact form. It means that we divide the Hamiltonian (1.10) into two parts:

$$
\tilde{\mathcal{H}}=\tilde{\mathcal{H}}_{j}+\left(\tilde{\mathcal{H}}-\tilde{\mathcal{H}}_{j}\right)
$$

where the first term

$$
\tilde{\mathcal{H}}_{j}=\frac{1}{2} \sum_{n=1}^{2 S} \sum_{n^{\prime \prime}=1}^{2 S}\left(S_{j}^{z}\right)^{n} \sum_{j^{\prime \prime} \in j} U_{j j^{\prime \prime}}^{n n^{\prime \prime}}\left(S_{j^{\prime \prime}}^{z}\right)^{n^{\prime \prime}}-\sum_{n=1}^{2 S}\left(h_{j}^{(n)}+\mu_{j}^{(n)}\right)\left(S_{j}^{z}\right)^{n}
$$

corresponds to the considered site $j$ and the second one formally excludes the interactions referring to the site $j$. Then, the formula (2.1) can be rewritten as

$$
\frac{\operatorname{Tr}\left[\left(S_{j}^{z}\right)^{n} \exp (-\beta \tilde{\mathcal{H}})\right]}{\operatorname{Tr} \exp (-\beta \tilde{\mathcal{H}})}=\frac{\operatorname{Tr}\left\{\left[\frac{\operatorname{Tr}\left[\left(S_{\tilde{j}}^{z}\right)^{n} \exp (-\beta \tilde{\mathcal{H}})\right]}{\operatorname{Tr} \exp (-\beta \tilde{\mathcal{H}})}\right] \exp \left(-\beta\left(\tilde{\mathcal{H}}-\tilde{\mathcal{H}}_{j}\right)\right)\right\}}{\operatorname{Tr} \exp \left(-\beta\left(\tilde{\mathcal{H}}-\tilde{\mathcal{H}}_{j}\right)\right)}
$$

hence

$$
\left\langle\left(S_{j}^{z}\right)^{n}\right\rangle=\left\langle F_{n}\left(X_{j}^{(n)}\right)\right\rangle
$$

with

$$
X_{j}^{(n)}=\sum_{n^{\prime \prime}=0}^{2 S} \sum_{j^{\prime \prime} \in j} U_{j j^{\prime \prime}}^{n n^{\prime \prime}}\left(S_{j \prime \prime}^{z}\right)^{n^{\prime \prime}}
$$

which are the parameters of the effective field with respect to the trace over $j$, but which become the operators with respect to the states for $j^{\prime} \neq j$. We rewrite the Eq. (2.2) into the form

$$
\left\langle\left(S_{j}^{z}\right)^{n}\left(S_{j^{\prime}}^{z}\right)^{n^{\prime}}\right\rangle=\left\langle\left(S_{j^{\prime}}^{z}\right)^{n^{\prime}} F_{n}\left(X_{j}^{(n)}\right)\right\rangle
$$

which leads together with (2.6) to the set of equations for different kinds of the pair correlations. The relations include the correlations of higher orders, i.e. for three, four or more operators $\left(S_{j}^{z}\right)^{n}$. Therefore, we need to create new equations for the three-site correlations, four-site correlations, etc., in order to solve the infinite chain of equations, or we need to cut this chain in a proper place and to split the correlations of higher orders into the pair correlations. Usually, the second way is used and then the equations for the average values of the order parameter as well as for the average values of the pair correlations are sufficient.

In order to obtain the set of equations in their form for the pair correlations the function $F_{n}\left(X_{j}^{(n)}\right)$ is not necessary to be known, because it is quite sufficient to express the average value of $F_{n}\left(X_{j}^{(n)}\right)$ by the following formula:

$$
\left\langle F_{n}\left(X_{j}^{(n)}\right)\right\rangle=\int_{-\infty}^{\infty} \mathrm{d} x F_{n}(x)\left\langle\delta\left(x-X_{j}^{(n)}\right)\right\rangle
$$

Next, using the properties of the $\delta$ function: 


$$
\begin{aligned}
& \left\langle F_{n}\left(X_{j}^{(n)}\right)\right\rangle=\frac{1}{2 \pi} \int_{-\infty}^{\infty} \mathrm{d} x F_{n}(x) \\
& \times \int_{-\infty}^{\infty} \mathrm{d} t \exp (\mathrm{i} x t)\left\langle\prod_{j^{\prime \prime} \in j, n^{\prime \prime}=0}^{2 S} \exp \left(-\mathrm{i} t U_{j j^{\prime \prime}}^{n n^{\prime \prime}}\left(S_{j^{\prime \prime}}^{z}\right)^{n^{\prime \prime}}\right)\right\rangle
\end{aligned}
$$

and the relation

$$
\exp \left(-\mathrm{i} t U_{j j^{\prime \prime}}^{n n^{\prime \prime}}\left(S_{j^{\prime \prime}}^{z}\right)^{n^{\prime \prime}}\right)=\sum_{k=0}^{2 S} A_{k}\left(t U_{j j^{\prime \prime}}^{n n^{\prime \prime}}\right)\left[\left(S_{j^{\prime \prime}}^{z}\right)^{n^{\prime \prime}}\right]^{k}
$$

we can write the set of equations for the pair correlations in their explicit form which depends on the crystallographic lattice, i.e. the distribution of the nearest neighbours $j^{\prime \prime}$ with respect to the site $j$. Thus, it is not possible to discuss the problem in a general way but we need to assume details concerning the structure.

From the general point of view we can remark that the equations depend on the numerical coefficients which can be calculated only in the particular cases, it means for the physical situations exactly defined.

In order to illustrate this method we consider here the case of adlayer which contains one kind of atoms distributed at the square lattice. The degree of the surface coverage $\theta$ is assumed to be less than 1 , so any site can be occupied or unoccupied. This particular chemisorption problem can be formally written as a problem of magnetism for spin $S=1 / 2$. In this case the pseudospin operator is expressed by:

$$
S_{j}^{z}=\sigma_{j}(1)-1 / 2=1 / 2-\sigma_{j}(0)
$$

and the Hamiltonian (2.4) takes its form:

$$
\tilde{\mathcal{H}}=-\left(h_{j}^{(1)}+\mu_{j}^{(1)}\right) S_{j}^{z}+\frac{1}{2} S_{j}^{z} \sum_{j^{\prime \prime} \in j} U_{j j^{\prime \prime}}^{11} S_{j^{\prime \prime}}^{z}
$$

Taking into account (2.1) and (2.5) we obtain the following expression for the average value of operator $S_{j}^{z}$ :

$$
\left\langle S_{j}^{z}\right\rangle=\left\langle\frac{1}{2} \operatorname{tgh}\left[\frac{1}{2} \beta\left(h_{j}^{(1)}+\mu_{j}^{(1)}\right)-\frac{1}{4} \beta X_{j}^{(1)}\right]\right\rangle,
$$

With regard to the identity (2.6), it means that in this case

$$
F_{1}\left(X_{j}^{(1)}\right)=\frac{1}{2} \operatorname{tgh}\left[\frac{1}{2} \beta\left(h_{j}^{(1)}+\mu_{j}^{(1)}\right)-\frac{1}{4} \beta X_{j}^{(1)}\right] .
$$

We have to calculate the average value of the above expression, and for this 
purpose we use the Eqs. (2.10) and (2.11) which in this case become:

$$
\begin{aligned}
& \left\langle F_{1}\left(X_{j}^{(1)}\right)\right\rangle=\frac{1}{2 \pi} \int_{-\infty}^{\infty} \mathrm{d} x F_{1}(x) \int_{-\infty}^{\infty} \mathrm{d} t \exp (\mathrm{i} x t) \\
& \times\left\langle\prod_{j^{\prime \prime} \in j} \exp \left(-\mathrm{i} t U_{j j^{\prime \prime}}^{11}\left(S_{j^{\prime \prime}}^{z}\right)\right)\right\rangle, \\
& \exp \left(-\mathrm{i} t U_{j j^{\prime \prime}}^{11}\left(S_{j^{\prime \prime}}^{z}\right)\right)=\left[\cos \left(t U_{j j^{\prime \prime}}^{11}\right)-\mathrm{i} S_{j}^{z} \sin \left(t U_{j j^{\prime \prime}}^{11}\right)\right]
\end{aligned}
$$

with $j^{\prime \prime}=1,2,3,4$.

Taking into account (2.6) and assuming that for each pair of sites $j$ and $j^{\prime \prime}$ the pair interaction potentials are the same $U_{j j^{\prime \prime}}^{11}=U$, we obtain:

$$
\begin{aligned}
& \left\langle S_{j}^{z}\right\rangle=a_{0}+4 a_{1}\left\langle S_{1}^{z}\right\rangle+a_{2}\left[4\left\langle S_{1}^{z} S_{2}^{z}\right\rangle+2\left\langle S_{1}^{z} S_{2}^{z}\right\rangle\right]+4 a_{3}\left\langle S_{1}^{z} S_{2}^{z} S_{3}^{z}\right\rangle \\
& +a_{4}\left\langle S_{1}^{z} S_{2}^{z} S_{3}^{z} S_{4}^{z}\right\rangle
\end{aligned}
$$

where

$$
a_{k}=(-\mathrm{i})^{k} \frac{1}{2 \pi} \int_{-\infty}^{\infty} \mathrm{d} x F_{1}(x) \int_{-\infty}^{\infty} \mathrm{d} t \exp (\mathrm{i} x t) \cos ^{4-k}(U t) \sin ^{k}(U t) .
$$

Analogously to the above presented procedure, using the Eq. (2.8), we can obtain the following equation for average value of $S_{j}^{z} S_{j}^{z}$ :

$$
\begin{aligned}
& \left\langle S_{j}^{z} S_{j^{\prime}}^{z}\right\rangle=a_{0}\left\langle S_{1}\right\rangle+a_{1}\left[\left\langle S_{1}^{z}\right\rangle+2\left\langle S_{1}^{z} S_{2}^{z}\right\rangle+\left\langle S_{1}^{z} S_{3}^{z}\right\rangle\right] \\
& +a_{2}\left[2\left\langle S_{1}^{z} S_{2}^{z}\right\rangle+\left\langle S_{1}^{z} S_{3}^{z}\right\rangle+3\left\langle S_{1}^{z} S_{2}^{z} S_{3}^{z}\right\rangle\right] \\
& +a_{3}\left[\left\langle S_{1}^{z} S_{2}^{z} S_{3}^{z}\right\rangle+\left\langle S_{1}^{z} S_{2}^{z} S_{3}^{z} S_{4}^{z}\right\rangle\right]+a_{4}\left\langle S_{1}^{z} S_{2}^{z} S_{3}^{z} S_{4}^{z}\right\rangle
\end{aligned}
$$

in the case when $j$ and $j^{\prime}$ are the nearest neighbour sites (Fig. 1a) and

$$
\begin{aligned}
& \left\langle S_{j}^{z} S_{j^{\prime}}^{z}\right\rangle=\left\langle S_{j}^{z} F\left(\sum_{l \in j^{\prime}} S_{l}^{z}\right)\right\rangle=a_{0}\left\langle S_{1}^{z}\right\rangle+a_{1}\left[2\left\langle S_{1}^{z} S_{j}^{z}\right\rangle+2\left\langle S_{3}^{z} S_{j}^{z}\right\rangle\right] \\
& +a_{2}\left[\left\langle S_{3}^{z} S_{4}^{z} S_{j}^{z}\right\rangle+2\left\langle S_{2}^{z} S_{3}^{z} S_{j}^{z}\right\rangle+\left\langle S_{1}^{z} S_{2}^{z} S_{j}^{z}\right\rangle+2\left\langle S_{1}^{z} S_{3}^{z} S_{j}^{z}\right\rangle\right] \\
& +a_{3}\left[2\left\langle S_{1}^{z} S_{2}^{z} S_{3}^{z} S_{j}^{z}\right\rangle+2\left\langle S_{2}^{z} S_{3}^{z} S_{4}^{z} S_{j}^{z}\right\rangle\right]+a_{4}\left\langle S_{1}^{z} S_{2}^{z} S_{3}^{z} S_{4}^{z} S_{j}^{z}\right\rangle
\end{aligned}
$$

in the case when we consider the next nearest neighbour sites (Fig. 1b). The coefficients $a_{k}(k=0,1,2 \ldots)$ are still given by the Eq. (2.19).

Let us define now for the sake of convenience:

$$
\begin{aligned}
& X_{1}=\left\langle S_{j}^{z}\right\rangle, \\
& X_{2}=\left\langle S_{j}^{z} S_{j^{\prime}}^{z}\right\rangle, \\
& X_{3}=\left\langle S_{j}^{z} S_{j^{\prime}}^{z} S_{j^{\prime \prime}}^{z}\right\rangle, \quad \text { etc. }
\end{aligned}
$$



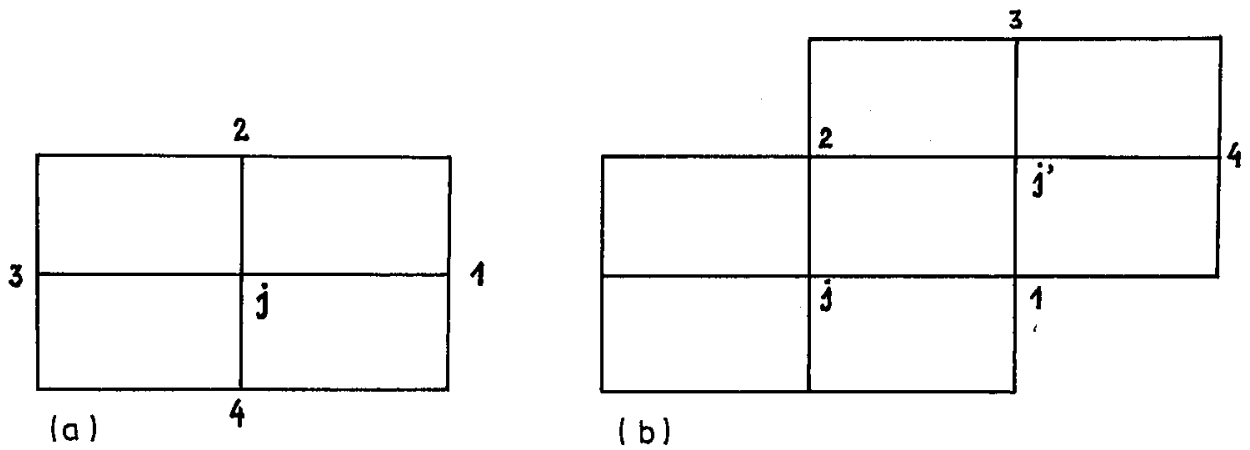

Fig. 1. The pseudospin positions in square lattice when sites $j$ and $j^{\prime}$ are: (a) the nearest neighbour sites $\left(j^{\prime}=1,2,3,4\right)$; (b) the next nearest neighbour sites $(l=$ $\left.1,2,3,4 \in j^{\prime}\right)$.

and in the first step of approximation we will put in the Eqs. (2.18) and (2.20) $X_{n} \approx X_{1}^{n}$ where $n=1,2,3, \ldots$ :

$$
\begin{aligned}
& X_{1}=a_{0}+4 a_{1} X_{1}+6 a_{2} X_{1}^{2}+4 a_{3} X_{1}^{3}+a_{4} X_{1}^{4}, \\
& X_{2}=b_{1} X_{1}+3 b_{2} X_{1}^{2}+3 b_{3} X_{1}^{3}+b_{4} X_{1}^{4},
\end{aligned}
$$

with

$$
\begin{aligned}
& b_{1}=a_{0}+a_{1}, \\
& b_{2}=a_{1}+a_{2}, \\
& b_{3}=a_{2}+a_{3}, \\
& b_{4}=a_{3}+a_{4} .
\end{aligned}
$$

With this approximation we can calculate the correlation $\gamma_{j 1}=X_{2}-\left(X_{1}\right)^{2}$ :

$$
\gamma_{j 1}=b_{1} X_{1}+\left(3 b_{2}-1\right) X_{1}^{2}+3 b_{3} X_{1}^{3}+b_{4} X_{1}^{4},
$$

where the mean value of pseudospin $X_{1}$ can be expressed in terms of the degree of the surface coverage $\theta$, namely $X_{1}=\theta-1 / 2$ and is calculated from the Eq. (2.23).

In order to calculate $\gamma_{j 1}$ more precisely and to have the possibility of calculating also the correlations of father sites $\gamma_{j 2}, \gamma_{j 4}$, etc. (Fig. 2) we will use the relations between cumulants defined in the following way:

$$
\begin{aligned}
& \left\langle S_{j}^{z}\right\rangle_{\mathrm{c}}=\left\langle S_{j}^{z}\right\rangle=X_{1}, \\
& \left\langle S_{j}^{z} S_{j^{\prime}}^{z}\right\rangle=\left\langle S_{j}^{z} S_{j^{\prime}}^{z}\right\rangle_{\mathrm{c}}+\left\langle S_{j}^{z}\right\rangle_{\mathrm{c}}\left\langle S_{j^{\prime}}^{z}\right\rangle_{\mathrm{c}}=\gamma_{j j^{\prime}}+\left(X_{1}\right)^{2}
\end{aligned}
$$




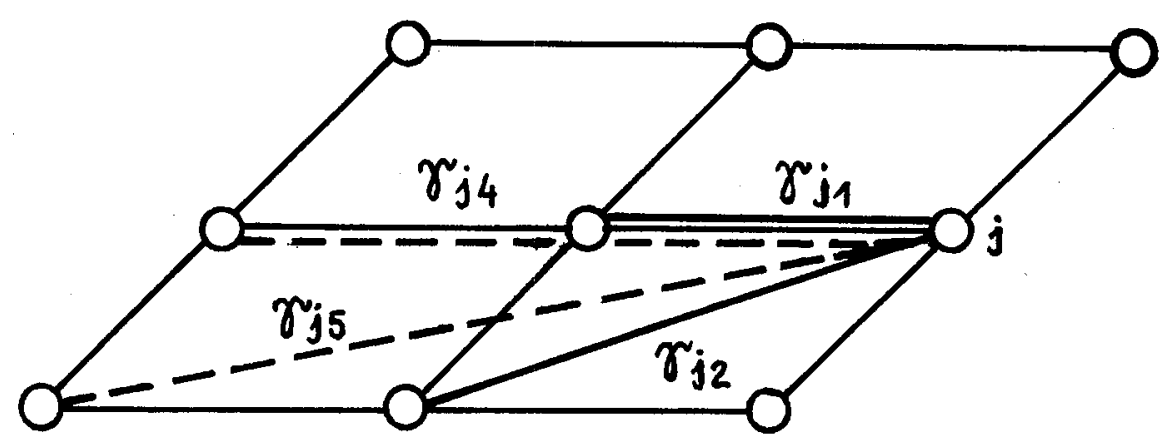

Fig. 2. The positions of pseudospin pairs in square lattice for correlation functions $\gamma_{j k}$ where $k$ is a distance of lattice sites.

and the multi-correlation functions. From these general relations presented in [3] we have the following approximated relations:

$$
\begin{aligned}
& \left\langle S_{j}^{z} S_{j^{\prime}}^{z} S_{j^{\prime \prime}}^{z}\right\rangle=X_{1}^{3}+X_{1}\left(\gamma_{j j^{\prime}}+\gamma_{j j^{\prime \prime}}+\gamma_{j^{\prime} j^{\prime \prime}}\right), \\
& \left\langle S_{j}^{z} S_{j^{\prime}}^{z} S_{j^{\prime \prime}}^{z} S_{j^{\prime \prime \prime}}^{z}\right\rangle=X_{1}^{4}+X_{1}^{2}\left(\gamma_{j j^{\prime}}+\gamma_{j j^{\prime \prime}}+\ldots+\gamma_{j^{\prime \prime} j^{\prime \prime \prime}}\right) \\
& +\gamma_{j j^{\prime}} \gamma_{j^{\prime \prime} j^{\prime \prime \prime}}+\gamma_{j j^{\prime \prime}} \gamma_{j^{\prime} j^{\prime \prime \prime}}+\gamma_{j j^{\prime \prime \prime}} \gamma_{j^{\prime} j^{\prime \prime}}
\end{aligned}
$$

and so on, where cumulants of order higher than two are neglected as small enough. As it is seen from above in the Eqs. (2.18), (2.20) and (2.21) can appear the correlations of father sites. So, these equations form the set of an infinite number of equations solved by the iteration procedure starting with $X_{1}$ and $\gamma_{j 1}$ calculated from (2.23) and (2.25):

$$
\begin{aligned}
X_{1} & =f\left(X_{1}, \gamma_{j 2}, \gamma_{j 4}\right), \\
\gamma_{j 1} & =f\left(X_{1}, \gamma_{j 2}, \gamma_{j 4}\right) ; \\
\gamma_{j 2} & =f\left(X_{1}, \gamma_{j 2}, \gamma_{j 4}, \gamma_{j 1}, \gamma_{j 5}\right), \\
\gamma_{j 4} & =f\left(X_{1}, \gamma_{j 2}, \gamma_{j 4}, \gamma_{j 1}, \gamma_{j 5}, \gamma_{j 9}\right) .
\end{aligned}
$$

We can calculate as many correlations as we need cutting this iteration in the proper place. 


\section{Constant coupling approximation}

The static pair correlations may be calculated on the basis of the effective field whose distribution is associated with the local deviation of the order parameter from its average value. The idea is related to the description of the fluctuations in the mean density of elements by the pair correlation functions introduced between clusters of elements in macroscopically small cells, on the condition that the linear dimension of the clusters remains small compared to the range of correlation.

There are two assumptions which allow us to express the correlation function (1.9) by means of the effective field, namely: 1 ) the correlations between elements at the sites $j$ and $j^{\prime}$ may be replaced by the correlations between clusters, and 2 ) the statistical average of the product of fluctuations may be replaced by the product of statistical a verages. It means:

$$
\gamma_{j j^{\prime}}^{n n^{\prime}}=\overline{\delta\left(S_{j}^{z}\right)^{n}} \overline{\delta\left(S_{j^{\prime}}^{z}\right)^{n^{\prime}}}
$$

where $\delta\left(S_{j}^{z}\right)^{n}=\left(S_{j}^{z}\right)^{n}-\left\langle\left(S_{j}^{z}\right)^{n}\right\rangle$ denotes the local deviation of the operator $S_{j}^{z}$ from its statistical average value. The pair correlations calculated on the basis of relation (3.1) are valid at all distances with an accuracy depending on the correctness of the introduced approximations by the assumptions mentioned above.

The symmetry conditions allow us to consider the origin of fluctuations independently of its position in the system. Thus, all the sites are equivalent and therefore we can assume $j=0$ for a site $j$ given arbitrarily. Then the distance $j^{\prime}-j=r$ denotes the position of the correlated elements.

In order to express the correlation functions in terms of the local effective fields we consider the solutions for the Hamiltonian (1.6) in the constant coupling approximation where the thermodynamic properties of a system of $N$ elements are described by the properties of a statistical pair of the nearest neighbouring sites [4]. In particular, the free energy $F$ of the system is given by the free energies of pairs, i.e.:

$$
F=\frac{1}{2} N z\left(E^{(2)}-T S^{(2)}\right),
$$

where $N z / 2$ is the number of pairs of nearest neighbouring sites, $z$ is the coordination number and $E^{(2)}$ is the internal energy of a pair:

$$
E^{(2)}=\operatorname{Tr}\left(\rho^{(2)} H^{(2)}\right),
$$

while $S^{(2)}$ denotes the pair entropy:

$$
S^{(2)}=-k\left[\sum_{v=0}^{3} f_{v} \ln f_{v}+\frac{z-1}{z} \sum_{n=1}^{2 S} \ln \left\{4 \frac{\left(1-\left(S^{*}\right)^{n}\right)^{\left(S^{*}\right)^{n}-1}}{\left(1+\left(S^{*}\right)^{n}\right)^{\left(S^{*}\right)^{n}+1}}\right\}\right]
$$

with $k$ standing for the Boltzmann constant.

The pair density operator:

$$
\rho^{(2)}=\exp \left(-\beta \mathcal{H}^{\mathrm{e}}\right)\left[\operatorname{Tr} \exp \left(-\beta \mathcal{H}^{\mathrm{e}}\right)\right]^{-1}
$$


is determined on the basis of the so-called effective Hamiltonian:

$$
\mathcal{H}^{\mathrm{e}}=\sum_{n=1}^{2 S} \sum_{n^{\prime}=1}^{2 S} U_{j j^{\prime}}^{n n^{\prime}}\left(S_{j}^{z}\right)^{n}\left(S_{j^{\prime}}^{z}\right)^{n^{\prime}}-\sum_{n=1}^{2 S}\left[H_{j}^{(n)}\left(S_{j}^{z}\right)^{n}+H_{j^{\prime}}^{(n)}\left(S_{j^{\prime}}^{n}\right)^{n}\right]
$$

containing the effective field $H_{j}^{(n)}$ which corresponds to the molecular field when a statistical pair of nearest neighbours is embedded in it.

The Hamiltonian $H^{(2)}$ is defined by:

$$
\begin{aligned}
& H^{(2)}=\sum_{n=1}^{2 S} \sum_{n^{\prime}=1}^{2 S} U_{j j^{\prime}}^{n n^{\prime}}\left(S_{j}^{z}\right)^{n}\left(S_{j^{\prime}}^{z}\right)^{n^{\prime}} \\
& -z^{-1} \sum_{n=1}^{2 S}\left[h_{j}^{(n)}\left(S_{j}^{z}\right)^{n}+h_{j^{\prime}}^{(n)}\left(S_{j^{\prime}}^{n}\right)^{n}\right]
\end{aligned}
$$

so that the Hamiltonian (1.6) may be written as $\mathcal{H}=\frac{1}{2} N z H^{(2)}$.

The long range order parameters $\left(S^{*}\right)^{n}$ which appear in the entropy formula (3.4) are defined by:

$$
\left(S^{*}\right)^{n}=\operatorname{Tr}\left[\rho^{(2)}\left(\left(S_{j}^{z}\right)^{n}+\left(S_{j}^{z}\right)^{n}\right)\right]
$$

and they become independent of the sites $j$ and $j^{\prime}$ because of the statistical character of the considered pair in the case of equilibrium conditions assuring a homogeneous distribution of the effective field. Taking into account the eigenvalues $\mathcal{E}_{v}$ of the effective Hamiltonian (3.6) we can define the quantities:

$$
f_{v}=\frac{\exp \left(-\beta \mathcal{E}_{v}\right)}{\sum_{v} \exp \left(-\beta \mathcal{E}_{v}\right)}
$$

which allow us to express the parameters $(3.8)$ by $\left(S^{*}\right)^{1}=f_{1}-f_{3}$ as well as the free energy (3.2) in terms of the eigenvalues of the effective Hamiltonian which contains the pair interaction in its exact form.

The application of the equations in the constant coupling approximation to non-equilibrium conditions is accomplished by introducing into the effective Hamiltonian (3.6) terms depending on the fluctuations (3.1) of the effective field [4]. The considerations connected with the system described by means of the grand canonical ensemble require to take into account for the effective Hamiltonian (3.6) additional terms corresponding to the chemical potentials, i.e. to replace the Hamiltonian (1.6) by (1.10).

For the purposes of the approach to the correlation functions in the system with the topological disorder fixed by the degree of coverage we consider the effective Hamiltonian in the following form:

$$
\tilde{\mathcal{H}}_{r}^{\mathrm{e}}=\mathcal{H}_{r}^{\mathrm{e}}-\mu_{r} S_{r}^{z}-\mu_{r+\delta} S_{r+\delta}^{z}
$$


where

$$
\begin{aligned}
& \mathcal{H}_{r}^{\mathrm{e}}=\sum_{n=1}^{2 S} \sum_{n^{\prime}=1}^{2 S} U_{r r+\delta}^{n n^{\prime}}\left(S_{r}^{z}\right)^{n}\left(S_{r+\delta}^{z}\right)^{n^{\prime}} \\
& -\sum_{n=1}^{2 S}\left[H_{r}^{(n)}\left(S_{r}^{z}\right)^{n}+H_{r+\delta}^{(n)}\left(S_{r+\delta}^{n}\right)^{n}\right]
\end{aligned}
$$

which should replace the Hamiltonian $\mathcal{H}^{\mathrm{e}}$ appearing in the Eqs. (3.3)-(3.5), (3.8) and (3.9). Then the Hamiltonian $H^{(2)}$ is replaced by:

$$
\begin{aligned}
& \tilde{H}^{(2)}=\sum_{n=1}^{2 S} \sum_{n^{\prime}=1}^{2 S} U_{r r+\delta}^{n n^{\prime}}\left(S_{r}^{z}\right)^{n}\left(S_{r+\delta}^{z}\right)^{n^{\prime}} \\
& -z^{-1} \sum_{n=1}^{2 S}\left[\left(h_{r}^{(n)}+\mu_{r}\right)\left(S_{r}^{z}\right)^{n}+\left(h_{r+\delta}^{(n)}+\mu_{r+\delta}\right)\left(S_{r+\delta}^{z}\right)^{n}\right]
\end{aligned}
$$

so that the Hamiltonian (1.10) may be now written as $\tilde{H}=\frac{1}{2} N z \tilde{H}^{(2)}$, respectively.

The effective fields $h_{r}^{(n)}$ represent local fluctuations (3.1) and lead to the results of local character for all the properties of the system. In particular, the order parameters (3.8) become inhomogeneous in general, namely:

$$
\left(S_{r}^{*}\right)^{n}=\operatorname{Tr}\left[\tilde{\rho}^{(2)}\left(\left(S_{r}^{z}\right)^{n}+\left(S_{r+\delta}^{z}\right)^{n}\right)\right] .
$$

Therefore, it is convenient to introduce the short range order parameters:

$$
\left(s_{r}^{*}\right)^{n}=\operatorname{Tr}\left[\tilde{\rho}^{(2)}\left(\left(S_{r}^{z}\right)^{n}-\left(S_{r+\delta}^{z}\right)^{n}\right)\right]
$$

which allow us to determine the average values:

$$
\left\langle\left(S_{r}^{z}\right)^{n}\right\rangle=\frac{1}{2}\left[\left(S_{r}^{*}\right)^{n}+\left(s_{r}^{*}\right)^{n}\right]
$$

with respect to the nearest neighbourhood appearing by the summation over $\delta$.

It is worthwhile to notice that the internal energy of a pair (3.3) calculated by means of the Hamiltonian (3.11) becomes the enthalpy due to the terms connected with chemical potentials and then the free energy (3.2) becomes the Gibbs potential:

$$
G=\frac{1}{2} \sum_{r}^{N} \sum_{\delta}^{z}\left(E_{r, r+\delta}^{(2)}-T S_{r, r+\delta}^{(2)}\right)
$$

respectively.

In order to calculate the behaviour of the effective fields $h_{r}^{(n)}$ we apply the variational procedure to the local Gibbs potential increase due to the appearance of fluctuations, namely:

$$
\Delta G_{r}=\sum_{\delta}^{z}\left(G_{r, r+\delta}-G_{r, r+\delta}^{0}\right)
$$


where $G_{r, r+\delta}^{0}$ denotes the Gibbs potential independent of $h_{r}^{(n)}$ and $G_{r, r+\delta}=$ $E_{r, r+\delta}^{(2)}-T S_{r, r+\delta}^{(2)}$ is found by the procedure described above.

The effective fields $h_{r}^{(n)}$ according to the Hamiltonian (3.10) appear in two sites $r$ and $r+\delta$, where $\delta$ refers to the nearest neighbours. We can pass to the continuous variable $r$ and expand $h_{r+\delta}^{(n)}$ in Taylor series retaining terms up to the second order. Then the Euler-Lagrange equation for the functional $\Delta G_{r}$ leads to the equations for the fluctuation of effective fields $h_{r}^{(n)}$ :

$$
\nabla \frac{\partial \Delta G_{r}}{\partial \nabla h_{r}^{(n)}}-\frac{\partial \Delta G_{r}}{\partial h_{r}^{(n)}}=0
$$

The solutions of (3.17) allow us to calculate the average values (3.14) which are connected with the quantities:

$$
\overline{\delta\left(S_{r}^{z}\right)^{n}}=\left\langle\left(S_{r}^{z}\right)^{n}\right\rangle-\left\langle\left(S_{r}^{z}\right)_{0}^{n}\right\rangle
$$

appearing in (3.1) and determining the correlation functions. The index 0 in (3.18) denotes the average value taken for $(3.10)$ with $h_{r}^{(n)}=0$. Finally, we obtain:

$$
\gamma_{0 r}^{n n^{\prime}}=\Gamma_{0}^{n}\left[\left\langle\left(S_{r}^{z}\right)^{n^{\prime}}\right\rangle-\left\langle\left(S_{r}^{z}\right)_{0}^{n^{\prime}}\right\rangle\right]
$$

where the constant $\Gamma_{0}^{n}$ is related to $h_{0}^{(n)}$ under some physical conditions at the origin of correlations and it can be treated as the parameter of the theory.

The formula (3.19) reflects the symmetry relations, which means that the correlations referring to an arbitrary site as their origin are equivalent, i.e. we can always assume $j^{\prime}=j+r$ and consider $j=0$ as the origin of the coordinate system for the site $j$ given arbitrarily.

We discuss the correlation functions for $S=1 / 2$ as an example in order to show the details and the effective form of correlations. In this case the eigenvalues $\mathcal{E}_{v}(v=0,1,2,3)$ of the effective Hamiltonian (3.10) are expressed by [4]:

$$
\begin{aligned}
& \mathcal{E}_{0}=-\frac{1}{4} U_{r r+\delta}^{11}+\frac{1}{2}\left[\left(U_{r r+\delta}^{11}\right)^{2}+(\delta \mu)^{2}\right]^{1 / 2}, \\
& \mathcal{E}_{2}=-\frac{1}{4} U_{r r+\delta}^{11}-\frac{1}{2}\left[\left(U_{r r+\delta}^{11}\right)^{2}+(\delta \mu)^{2}\right]^{1 / 2}, \\
& \mathcal{E}_{1}=\frac{1}{4} U_{r r+\delta}^{11}-\sum H-\frac{1}{2} \sum \mu, \\
& \mathcal{E}_{3}=\frac{1}{4} U_{r r+\delta}^{11}+\sum H+\frac{1}{2} \sum \mu,
\end{aligned}
$$

where

$$
\begin{aligned}
& \sum \mu \equiv \mu_{r+\delta}+\mu_{r} \\
& \delta \mu \equiv \mu_{r+\delta}-\mu_{r} \\
& \sum H \equiv H_{r+\delta}+H_{r}
\end{aligned}
$$


and belong to the eigenfunctions:

$$
\begin{aligned}
& |0\rangle=\varphi_{0} \cos \frac{\omega}{2}+\varphi_{2} \sin \frac{\omega}{2}, \\
& |1\rangle=\varphi_{2} \cos \frac{\omega}{2}-\varphi_{0} \sin \frac{\omega}{2}, \\
& |2\rangle=\varphi_{1}, \\
& |3\rangle=\varphi_{3}, \\
& \varphi_{0}=\frac{1}{\sqrt{2}}(|+-\rangle-|-+\rangle), \quad \varphi_{2}=\frac{1}{\sqrt{2}}(|+-\rangle+|-+\rangle), \\
& \varphi_{1}=|++\rangle, \quad \varphi_{3}=|--\rangle,
\end{aligned}
$$

where

$$
\sin \omega=\delta \mu\left[\left(U_{r r+\delta}^{11}\right)^{2}+(\delta \mu)^{2}\right]^{-1 / 2},
$$

while the functions $|++\rangle,|+-\rangle,|-+\rangle,|--\rangle$ stand for the basic system of the product eigenfunctions.

On the basis of the Eqs. (3.20)-(3.31) we can write $\Delta G_{r}$ and taking into account the Eq. (3.17) we find the following equation:

$$
\left(\nabla^{2}-\kappa_{1}^{2}\right) h_{r}^{1}=0
$$

for the molecular field $h_{r}^{1}$ which is associated with the correlation functions (3.19). The parameter $\kappa_{1}^{2}$ is given by the formula:

$$
\kappa_{1}^{2}=\frac{1}{a^{2}} \frac{2 z\left[\eta^{2}-(z-4) / z\right]}{k T / U_{r r+\delta}^{11}\left(\eta^{4}+\eta^{2}-3\right)-\left[\eta^{2}-(z-4) / z\right]},
$$

while the relation (3.19) leads to the formula:

$$
\gamma_{0 r}^{11}=\beta /\left(3+\eta^{2}\right) \Gamma_{0}^{1} h_{r}^{1},
$$

where $\eta=\exp \left(1 / 2 \beta U_{r r+\delta}^{11}\right)$.

The solution of the Eq. (3.32) is the well-known Ornstein-Zernike function when the spherical symmetry is assumed as the boundary conditions for the solutions of Eq. (3.32). The Fourier transform of this function is identical with that obtained within the molecular field approximation with respect to the $q$ dependence while the coefficient $\kappa_{1}$ is slightly different as compared with the corresponding factor in MFA.

However, in the case of the constant coupling approach the solution for the correlation functions can be considered in a more general form taking various assumptions which concern the symmetry of the correlations. When the symmetry is assumed to be identical with the crystallographic symmetry of a sample the solution of Eq. (3.32) can be found in the form reflecting the crystal symmetry (cf. the behaviour of magnetic correlations discussed in [4]). The consequence is quite important because of the behaviour of the Fourier transform as well as the 
cross section for the incoherent scattering of electrons. Therefore, it seems to us very interesting to study the dependence of the correlations on the crystallographic structures and to compare them with the experimental data which should be sufficently sensitive to distinguish between different correlation symmetries.

\section{Symmetry of the correlation functions}

The variational principle applied to the calculation of the best distribution of the correlations in the considered system shows that the correlations are determined by the differential equation whose symmetry remains to be defined under additional conditions. This fact allows us to distinguish between the fluctuation-dissipation theorem illustrated by the molecular field approximation [1] and variational principle leading to the description directly in the lattice space. It seems to be quite well justified that the natural conditions for the correlation symmetry are given in this case by the symmetry of the crystallographic lattice at least for the distance of the nearest neighbours. Of course, the spherical symmetry of correlations for long distances is more probable when the isotropic medium is considered. The additional advantage of the crystal symmetry reflected by the short-distance correlations is connected with the finite value of the autocorrelations while the spacial correlations lead to the divergence at the origin of correlations.

The assumption of the crystal lattice symmetry correlations was discussed in connection with the magnetic moment fluctuations [4] and their consequences yielding the cross section of critical scattering in the elastic approximation. A comparison between the calculations and experimental results for the magnetic critical scattering of neutrons and particularly ferroelectric critical scattering of $\mathrm{X}$-rays shows that some features of the behaviour of critical scattering intensities can be explained in a natural way when the correlation symmetry is introduced. Thus, the analogy between the considered phenomena suggests that the correlation symmetry should also play an essential role in the case explaining the behaviour of the diffuse LEED intensities.

In a general case we can solve the Eq. (3.32) in the form:

$$
\begin{aligned}
& \gamma(x, y, z)=\gamma_{0} \exp \left[-\kappa_{1} f(x, y, z)\right] \\
& f(x, y, z)>0
\end{aligned}
$$

with respect to the boundary conditions assuring the crystal lattice symmetry by a proper choice of the function $f(x, y, z)$. In particular, the function $f(x, y, z)$ was found for several structures, namely [5]:

$$
f(x, y, z)=\alpha|x|+\beta|y|+\gamma|z|
$$

for simple orthorombic lattice $\left(\alpha^{2}+\beta^{2}+\gamma^{2}=1\right)$ [4], as well as

$$
f(x, y)=2|x+y|+2|x-y|
$$

for body centered cubic lattice and

$$
f(x, y)=|x+y|+|x-y|+2|x|+2|y|
$$


for face centered cubic lattice. The case $\alpha^{2}=\beta^{2}=\gamma^{2}=1 / 3$ in (4.2) corresponds to the simple cubic lattice.

Taking into account the method used by Urbaniak-Kucharczyk [6] in order to find the function $f(x, y, z)$ in particular cases we report now some steps of the general approach to this question confined to two dimensional lattices on which our considerations focus.

The function $f(x, y)$ should be function of coordinated $x, y$ only. Thus the Eq. (3.32) leads to the following equations:

$$
\begin{aligned}
& \left(\frac{\partial^{2}}{\partial x^{2}}+\frac{\partial^{2}}{\partial y^{2}}\right) f(x, y)=0, \\
& \left(\frac{\partial^{2} f(x, y)}{\partial x^{2}}\right)^{2}+\left(\frac{\partial^{2} f(x, y)}{\partial y^{2}}\right)^{2}=1
\end{aligned}
$$

which should be be satisfied by $f(x, y)$ as independent of $\kappa_{1}$.

In order to make the symmetry of correlation function for the short distance in comparison with a lattice constant correspond to the symmetry of a lattice, the correlation function should be invariant for the passing from one nearest neighbour of reference site to another one by the rotation around the axis through this reference site. If we assume that the reference site corresponding to the fluctuation center is situated at $(0,0)$ site then the positions of its nearest neighbours are determined by the equations of planes passing through this reference site and its nearest neighbours. Moreover, taking into account the symmetry of neighbours for $x=-x$ and $y=-y$ we obtain the function $f(x, y)$ in the form

$$
f(x, y)=\alpha \sum_{i=1}^{n}\left|\xi_{i}\right|
$$

where $\xi_{i}$ comes from the equations of planes determining the positions of neighbouring atoms, $n$ is a number of the nearest neighbours of the reference site and $\alpha$ is a parameter which can be calculated from (4.6).

The problem of the correlation symmetry also appears in the cumulant average approach to the two-site correlations. It is worthwhile to notice that the problem mentioned above in the last case is even more evident and natural. The correlation functions calculated on the basis of the set of proper equations show the deviations from the spherical symmetry without any assumptions concerning the symmetry of correlations. The results reported for Ising ferromagnets [7] indicate that the symmetry is related to the crystal lattice. It is seen that for very small distances, measured in lattice constant $a$, the curves corresponding to various crystail', graphic directions become quite different. The magnitude of their splitting is maxiinal at the critical temperature and it vanishes for temperature tending to zero or to infinity. For any other temperature the correlation functions reflect the crystal lattice symmetry. 


\section{Final remarks}

The present paper brings some methods for the two-site correlation function descriptions. The methods are chosen representatively from the point of view of different methodological approaches and different levels of approximation. The models take into consideration the variational principle as well as the scheme for direct calculations of the thermodynamic averages with respect to two-site correlations given by the set of coupled equations. The methods presented in this part are complementary to those reported in the first part of review [1], where we considered the fluctuation-dissipation theorem and the Green function technique, both discussed on the molecular field level.

We can see that the different approaches lead to similar results in general but with evident differences for some features of correlations. In particular the behaviour of correlations with respect to temperature shows quite qualitative discrepances mostly below the critical temperature which depends on the coverage degree. It is worthwhile to note here that the correlations are considered in the case of grand canonical ensemble while the analogous calculations are performed usually in the case of canonical ensemble. This fact influences the critical behaviour and reflects the discovered situation that the correlation behaviour at the critical region depends strongly on the methods used for calculations.

Another question is connected with the symmetry of correlation functions. This feature is also discussed in the present review and it is qualitatively more coherent. First of all it is evident in the method of cumulant average applied to two-site correlations that these correlations are of non-spherical symmetry as natural properties of the system. This character of the symmetry can be easily interpreted on the basis of the variational principle whose solutions can take the form of an arbitrary symmetry. Their Fourier transforms represent then the specific behaviour of the intensities measured in the diffuse LEED experiments. This behaviour can be approached directly by the solutions of the fluctuation-dissipation theorem for which its Fourier transforms should reflect the symmetry but this condition is usually neglected. Only the comparison between various methods draws our attention to the fact that the condition in the symmetry does not touch the spherical one but it can be applied in a more general form. Thus it is possible for different approaches to be not contradictory.

The cumulant average approach seems to us to be the most convenient and precise to calculate the correlations and to discuss their properties. However, for the main purpose of the present considerations, i.e. for calculating the diffuse LEED intensities we need to know the Fourier transforms of the correlations and therefore we need a big number of correlations to be able to find their inverse quantities. From this point of view the method is tiring. Thus, we think that it is more useful to apply the molecular field approximation for temperatures above their critical value where the different methods are qualitatively and even quantitatively compared. 


\section{References}

[1] L. Wojtczak, I. Zasada, J.C. Le Bossé, J. Lopez, J. Rousseau, Acta Physicae Superficierum 1, (1990).

[2] J.C. Le Bossé, J. Lopez, J. Rousseau, I. Zasada, J. Phys. C: Solid State Phys. 21, 565 (1988).

[3] N. Matsudaira, J. Phys. Soc. Japan, 35, 1593 (1973).

[4] J. Kocinski, L. Wojtczak, Critical Scattering Theory, Elsevier Scientific Publishing Company, Amsterdam-Oxford-New York 1978.

[5] A. Urbaniak-Kucharczyk, Phys. Status Solidi B 93, K25 (1979).

[6] A. Urbaniak-Kucharczyk, Phys. Status Solidi B 109, K59 (1982).

[7] T. Balcerzak, J. Mielnicki, G. Wiatrowski, L. Wojtczak, Proceedings of the $V I^{\text {th }}$ Conference on Physics of Magnetism, Acta Magnetica, Supplement '90, 110. 